\title{
Poly-ADP-ribose polymerase inhibition protects against myocardial and endothelial reperfusion injury after hypothermic cardiac arrest
}

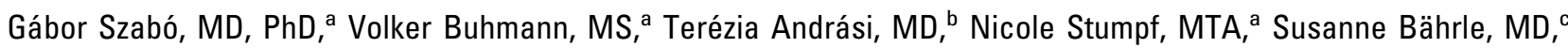
Violetta Kékesi, $\mathrm{PhD},{ }^{\mathrm{b}}$ Siegfried Hagl, MD, ${ }^{\mathrm{a}}$ Csaba Szabó, MD, PhD, ${ }^{\mathrm{d}}$ and Alexander Juhász-Nagy, MD, PhD ${ }^{\mathrm{b}}$

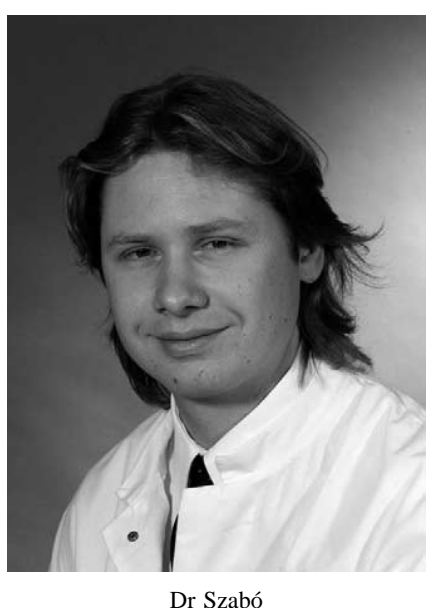

From the Department of Cardiac Surgery, University of Heidelberg, Heidelberg, Germany, ${ }^{\mathrm{a}}$ the Department of Cardiovascular Surgery, Semmelweis University Medical School, Budapest, Hungary, ${ }^{\mathrm{b}}$ the Department of Cardiology, Angiology and Pulmonology, University of Heidelberg, Heidelberg, Germany, ${ }^{\mathrm{c}}$ and Inotek Corporation, Beverly, Mass. ${ }^{d}$

Supported by grant "Forschungsschwerpunkt Transplantation," University of Heidelberg, and grant SFB414 of the German Research Foundation to G.S. and by a grant from the National Institutes of Health to C.S. (R01HL59266).

Read at the Eighty-second Annual Meeting of The American Association for Thoracic Surgery, Washington, DC, May 5-8, 2002.

Received for publication May 9, 2002; revisions requested June 14, 2002; revisions received July 18, 2002; accepted for publication Aug 14, 2002.

Address for reprints: Gábor Szabó, MD, $\mathrm{PhD}$, Department of Cardiac Surgery, Im Neuenheimer Feld 110, 69120 Heidelberg, Germany (E-mail: dzsi@ hotmail.com).

J Thorac Cardiovasc Surg 2003;126:651-8

Copyright $(2003$ by The American Association for Thoracic Surgery

$0022-5223 / 2003 \$ 30.00+0$

doi:10.1016/S0022-5223(02)73235-2
Objective: Free radical production and related cytotoxicity during ischemia and reperfusion might lead to DNA strand breakage, which activates the nuclear enzyme poly-ADP-ribose synthetase and initiates an energy-consuming and inefficient cellular metabolic cycle with transfer of the adenosine diphosphate-ribosyl moiety of nicotinamide adenine dinucleotide $\left(\mathrm{NAD}^{+}\right)$to protein acceptors. We investigated the effects of poly-ADP-ribose synthetase inhibition on myocardial and endothelial function during reperfusion in an experimental model of cardiopulmonary bypass.

Methods: Twelve anesthetized dogs underwent hypothermic cardiopulmonary bypass. After 60 minutes of hypothermic cardiac arrest, reperfusion was started after application of either saline vehicle (control, $\mathrm{n}=6)$ or PJ34 $(10 \mathrm{mg} / \mathrm{kg})$, a potent poly-ADP-ribose synthetase inhibitor $(n=6)$. Biventricular hemodynamic variables were measured by means of a combined pressure-volume conductance catheter, and the slope of the end-systolic pressure-volume relationships was calculated at baseline and after 60 minutes of reperfusion. Left anterior descending coronary blood flow, endothelium-dependent vasodilatation to acetylcholine, and endotheliumindependent vasodilatation to sodium nitroprusside were also determined.

Results: The administration of PJ34 led to a significantly better recovery of left and right ventricular systolic function $(P<.05)$ after 60 minutes of reperfusion. In addition, the inotropic adaptation potential of the right ventricle to an increased afterload was better preserved in the PJ34 group. Coronary blood flow was also significantly higher in the PJ34 group $(P<.05)$. Although the vasodilatory response to sodium nitroprusside was similar in both groups, acetylcholine resulted in a significantly higher increase in coronary blood flow in the PJ34 group $(P<.05)$.

Conclusions: Poly-ADP-ribose synthetase inhibition improves the recovery of myocardial and endothelial function after cardiopulmonary bypass with hypothermic cardiac arrest.

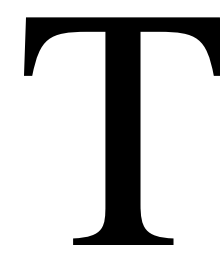

he majority of the cardiac surgical procedures done today are performed with aortic crossclamping and cardioplegic arrest. Despite improvements in cardioplegic techniques, ventricular dysfunction after cardioplegic arrest is a major cause of perioperative morbidity and mortality. ${ }^{1}$ Cardiocirculatory dysfunction associated with cardiopulmonary bypass (CPB) and cardiac arrest is often caused by decreased right ventricular function. ${ }^{2}$ This situation is aggravated by transient pulmonary hypertension occurring frequently after CPB as a result of endothelial injury, ${ }^{3}$ decreased nitric oxide, ${ }^{4}$ or increased thromboxane ${ }^{5}$ synthesis. Furthermore, coronary endothelial dysfunction might also complicate the early postoperative course. ${ }^{6}$

The activation of poly-ADP-ribose synthetase (PARP) is currently described to be a final common effector in various types of tissue injury, including systemic inflammation, circulatory shock, and ischemia and reperfusion. The genetic disrup- 
tion of the PARP pathway effectively protects against oxygen radical and nitric oxide toxicity in different cell cultures, such as pancreatic islet cells ${ }^{7}$ and thymocytes, ${ }^{8}$ and attenuates regional myocardial ${ }^{9}$ ischemia-reperfusion injury and global hypoxia-reoxygenation injury. ${ }^{10}$ Furthermore, the pharmacologic blockade of PARP also results in a protection against peroxynitrite injury in cardiomyocytes, ${ }^{11}$ endothelial cells, and fibroblasts ${ }^{9,12}$ and reduces myocardial infarct size in a regional ischemia model. ${ }^{13-15}$ It was also demonstrated that PARP inhibition leads to a significant improvement of endothelial function ex vivo in peroxynitrite-treated thoracic aortic rings ${ }^{16}$ and in isolated mesenteric arteries in the setting of splanchnic ischemia and reperfusion. ${ }^{17}$ In a recent rat heart transplant study, ${ }^{18}$ we showed energy depletion and histologically confirmed PARP activation after hypothermic cardiac preservation and reperfusion and improvement of functional recovery by means of PARP inhibition with the novel potent PARP inhibitor PJ34.

The aim of the present study was to test the hypothesis that PARP inhibition ${ }^{18}$ improves myocardial and endothelial function after hypothermic cardioplegic arrest and reperfusion in a clinically relevant canine model of CPB. On the basis of detailed biochemical and histologic data in previous studies, ${ }^{13,15,18}$ we focused on a functional analysis in the present experimental trial.

\section{Materials and Methods}

\section{Animals and Experimental Groups}

Twelve dogs (foxhounds) weighing 23 to $32 \mathrm{~kg}$ ( $26 \pm 3 \mathrm{~kg}$ ) were used in this experiment. All animals received humane care in compliance with the "Principles of Laboratory Animal Care" formulated by the National Society for Medical Research and the "Guide for the Care and Use of Laboratory Animals" prepared by the Institute of Laboratory Animal Resources and published by the National Institutes of Health (National Institutes of Health publication no. 86-23, revised 1996). The experiments were approved by the Ethical Committee of the Land Baden-Württemberg for Animal Experimentation. Six animals received $10 \mathrm{mg} / \mathrm{kg}$ PJ34, a novel, potent, water-soluble, phenanthridinone-derivative PARP inhibitor as a short infusion beginning 5 minutes before aortic declamping and continuing during the first 15 minutes of reperfusion. Six vehicle-treated animals served as control animals. The applied dose of PJ34 is based on our previous ischemia-reperfusion and pharmacokinetic studies. ${ }^{18}$

\section{Surgical Preparation and General Management}

The dogs were premedicated with propionylpromazine and anesthetized with a bolus of pentobarbital $(15 \mathrm{mg} / \mathrm{kg}$ initial bolus and then $0.5 \mathrm{mg} \times \mathrm{kg}^{-1} \times \mathrm{h}^{-1}$ administered intravenously), paralyzed with pancuronium bromide $(0.1 \mathrm{mg} / \mathrm{kg}$ bolus and then $0.2 \mathrm{mg} \times$ $\mathrm{kg}^{-1} \times \mathrm{h}^{-1}$ administered intravenously), and endotracheally intubated. The dogs were ventilated with a mixture of room air and oxygen (fraction of inspired oxygen of 60\%) at a frequency of 12 to 15 breaths/min and a tidal volume beginning at $15 \mathrm{~mL} \times$ $\mathrm{kg}^{-1} \times \min ^{-1}$. The settings were adjusted by maintaining arterial partial carbon dioxide pressure levels of between 35 and $40 \mathrm{~mm}$ $\mathrm{Hg}$. The femoral artery and vein were cannulated for recording aortic pressure and taking blood samples for the analysis of blood gases, electrolytes, and $\mathrm{pH}$. Basic intravenous volume substitution was carried out with Ringer solution at a rate of $1 \mathrm{~mL} \times \mathrm{kg}^{-1} \times$ $\min ^{-1}$. If necessary, the rate of volume substitution was modified according to the continuously controlled input-output balance to maintain cardiac output at baseline levels. According to the values of potassium, bicarbonate, and base excess, substitution included administration of potassium chloride and sodium bicarbonate $(8.4 \%)$. Neither catecholamines nor other hormonal or pressor substances were administered. Rectal temperature and standard peripheral electrocardiography were monitored continuously.

After left anterolateral thoracotomy in the fourth intercostal space, pericardiotomy, and isolation of the great vessels, a perivascular ultrasonic flow probe was attached to the ascendent aorta. A combined 6F Millar pressure-conductance catheter (Millar Instruments, Inc, Houston, Tex) with 6-mm spacing was inserted into the left ventricle through the apex. A second $6 \mathrm{~F}$ Millar pressureconductance catheter with 5-mm spacing was inserted into the right ventricle through the pulmonary artery. Aortic pressure and right atrial pressure were monitored with $5 \mathrm{~F}$ Millar catheter-tip manometers. Coronary blood flow was measured on the left anterior descending artery with a perivascular ultrasonic flow probe.

\section{CPB and Cardioplegia}

After systemic anticoagulation with sodium heparin (300 U/kg), the left subclavian artery was cannulated for arterial perfusion. The venous cannula was placed in the right atrium. The extracorporeal circuit consisted of a heat exchanger, a venous reservoir, a roller pump, and a membrane oxygenator primed with Ringer lactate solution $(1000 \mathrm{~mL})$ supplemented with heparin $(150 \mathrm{U} / \mathrm{kg})$ and 20 $\mathrm{mL}$ of sodium bicarbonate $(8.4 \%)$. After initiation of CPB, body temperature was cooled to $28^{\circ} \mathrm{C}$. After crossclamping of the aorta, the heart was arrested with $25 \mathrm{~mL} / \mathrm{kg}$ HTK solution $(15 \mathrm{mmol}$ of $\mathrm{NaCl}, 9 \mathrm{mmol}$ of $\mathrm{KCl}, 4 \mathrm{mmol}$ of $\mathrm{MgCl}_{2}, 6 \mathrm{mmol}$ of $\mathrm{H}_{2} \mathrm{O}, 18$ mmol of histidine hydrochloride monohydrate, $180 \mathrm{mmol}$ of histidine, $2 \mathrm{mmol}$ of tryptophan, $30 \mathrm{mmol}$ of mannitol, $0.015 \mathrm{mmol}$ of $\mathrm{CaCl}_{2}$, and $1 \mathrm{mmol}$ of potassium-hydrogen-2-oxopentandioat, $\mathrm{H}_{2} \mathrm{O}$ ). During cardiac arrest, the pump flow was set at $100 \mathrm{~mL} \times$ $\mathrm{kg}^{-1} \times \min ^{-1}$ to maintain perfusion pressure at greater than 35 to $40 \mathrm{~mm} \mathrm{Hg}$ at any time point, and alpha-stat management was applied.

Twenty minutes before crossclamp removal, rewarming was initiated. After 60 minutes of cardiac arrest, the aorta was declamped, and the heart was reperfused with normothermic blood in the bypass circuit. If necessary, ventricular fibrillation was counteracted with direct-current cardioversion of $40 \mathrm{~J}$. Ventilation was restarted with $100 \%$ oxygen. All animals were weaned from CPB without inotropic support 20 minutes after the release of the aortic crossclamp. Each animal underwent 90 minutes of CPB with 60 minutes of cardiac arrest.

\section{Data Acquisition and Analysis}

Heart rate and aortic pressure were monitored continuously.

Left ventricular systolic pressure, right ventricular systolic pressure (RVSP), maximum pressure development ( $\left.\mathrm{dP}_{\mathrm{d}} \mathrm{dt}_{\max }\right)$, left ventricular end-diastolic pressure, right ventricular end-diastolic 
TABLE 1. Hemodynamic variables

\begin{tabular}{|c|c|c|c|c|}
\hline & \multicolumn{2}{|c|}{ Baseline } & \multicolumn{2}{|c|}{$60 \mathrm{~min}$ of reperfusion } \\
\hline & Control & PJ34 & Control & PJ34 \\
\hline HR (beats/min) & $113 \pm 8$ & $122 \pm 7$ & $120 \pm 6$ & $127 \pm 9$ \\
\hline $\mathrm{MAP}(\mathrm{mm} \mathrm{Hg})$ & $103 \pm 10$ & $97 \pm 6$ & $75 \pm 7^{*}$ & $87 \pm 5 \dagger$ \\
\hline $\operatorname{LVSP}(\mathrm{mm} \mathrm{Hg})$ & $124 \pm 7$ & $122 \pm 7$ & $100 \pm 8^{*}$ & $109 \pm 9$ \\
\hline $\mathrm{dP} / \mathrm{dt}_{\max }(\mathrm{mm} \mathrm{Hg} / \mathrm{s})$ & $1715 \pm 137$ & $1764 \pm 98$ & $1313 \pm 108^{*}$ & $1578 \pm 118$ \\
\hline $\operatorname{LVEDP}(\mathrm{mm} \mathrm{Hg})$ & $10 \pm 2$ & $10 \pm 1$ & $11 \pm 3$ & $9 \pm 2$ \\
\hline $\mathrm{CO}(\mathrm{L} / \mathrm{min})$ & $2.48 \pm 0.40$ & $2.20 \pm 0.31$ & $1.99 \pm 0.32$ & $2.01 \pm 0.27$ \\
\hline Ees $(\mathrm{mm} \mathrm{Hg} / \mathrm{mL})$ & $5.5 \pm 1.9$ & $7.3 \pm 2.9$ & $2.6 \pm 0.3^{*}$ & $4.9 \pm 0.9 \dagger$ \\
\hline $\mathrm{dP} / \mathrm{dt} / \mathrm{EDV}\left(\mathrm{mm} \mathrm{Hg} \cdot \mathrm{s}^{-1} \cdot \mathrm{mL}^{-1}\right)$ & $57 \pm 7$ & $50 \pm 7$ & $53 \pm 5^{*}$ & $21 \pm 5 \dagger$ \\
\hline PRSW (kerg) & $75 \pm 4$ & $76 \pm 8$ & $56 \pm 6^{*}$ & $79 \pm 8 \dagger$ \\
\hline
\end{tabular}

All values are given as means \pm SEM. $H R$, Heart rate; MAP, mean aortic pressure; $L V S P$, left ventricular systolic pressure; $L V E D P$, left ventricular end-diastolic pressure; $C O$, cardiac output; $d P / d t / E D V$, slope of the $\mathrm{dP} / \mathrm{dt}_{\text {max }}$-end-diastolic volume relationship; $P R S W$, preload recruitable stroke work. ${ }^{*} P<.05$ versus baseline.

$\dagger P<.05$, control versus PJ34.

pressure (RVEDP), and cardiac output as the equivalent of aortic flow were monitored continuously. Stroke volume was calculated from the integrated flow signal and was used to calibrate the volume signal from the conductance catheter. Parallel conductance was estimated by means of rapid injection of $1 \mathrm{~mL}$ of hypertonic saline into the pulmonary artery or superior vena cava, respectively.

The volume signal provided by the conductance catheter was registered continuously (Sigma F5; Leycom, Leiden, The Netherlands) and computed with the Conduct PC software (Leycom). Left and right ventricular pressure-volume loops were constructed online. Vena caval occlusions were performed to obtain a series of loops for calculation of the slope (Ees) and intercept (V0) of the left and right ventricular end-systolic pressure-volume relationships. In addition, the slope of the left ventricular $\mathrm{dP} / \mathrm{dt}_{\max }$-enddiastolic volume relationship and preload recruitable stroke work were calculated as load-independent indices of myocardial contractility.

The pulmonary artery was constricted by tightening a snare around the pulmonary artery 3 to $4 \mathrm{~cm}$ distal to the right ventricular outflow tract to examine the adaptation potential of the right ventricle to an afterload increase. An increase in RVSP to approximately 35 and approximately $55 \mathrm{~mm} \mathrm{Hg}$ was achieved by means of progressive constriction of the pulmonary artery. Measurements were taken 10 minutes after pulmonary banding at both levels during steady-state conditions. After the second pulmonary banding level, the snare was loosened, and the dogs were then allowed to return to baseline steady state. If necessary, volume substitution was applied to keep cardiac output at a constant level during this protocol.

Coronary blood flow was measured with an ultrasonic flowmeter placed on the left anterior descending coronary artery. Coronary endothelium-dependent vasodilatation was assessed after intracoronary administration of a single bolus of acetylcholine $\left(10^{-7}\right.$ $\mathrm{mol} / \mathrm{L}$ ), and endothelium-independent vasodilatation was assessed after sodium nitroprusside administration $\left(10^{-4} \mathrm{~mol} / \mathrm{L}\right)$. The vasoresponse was expressed as the percentage change of baseline coronary blood flow.

All measurements were performed before CPB and after 60 minutes of reperfusion.
All values were expressed as means \pm SEM. The paired $t$ test was used to compare 2 means within groups. Individual means between the groups were compared by means of 1-way analysis of variance, followed by an unpaired $t$ test with Bonferroni correction for multiple comparisons and the post hoc Scheffe test.

\section{Results}

\section{Left Ventricular Function and Systemic Hemodynamics}

Hemodynamic variables are shown in Table 1. Baseline parameters did not differ between the groups and were within the physiologic range. Mean blood pressure during $\mathrm{CPB}$ was $58 \pm 6$ versus $65 \pm 7 \mathrm{~mm} \mathrm{Hg}$ in the control and PARP groups, respectively (not significant). After $60 \mathrm{~min}-$ utes of cardioplegic arrest and 60 minutes of reperfusion, heart rate did not change in either the control or the PJ34 group. Mean arterial pressure, left ventricular systolic pressure, and $\mathrm{dP} / \mathrm{dt}_{\max }$ decreased significantly $(P<.05)$ in the control group, whereas it remained unchanged in the PJ34 group. However, except mean arterial pressure, the hemodynamic parameters did not differ significantly between the groups. It should also be noted that cardiac output showed a clear decreasing tendency within the control group without reaching the level of significance (Table 1). The Ees, the slope of the left ventricular $\mathrm{dP} / \mathrm{dt}_{\max }$-end-diastolic volume relationship, and preload recruitable stroke work decreased significantly $(P<.05)$ to less than the baseline values in the control group and were significantly lower than those in the PJ34 group (Table 1 and Figure 1).

\section{Right Ventricular Function}

Right ventricular parameters, namely right ventricular endsystolic volume (RVESV), right ventricular end-diastolic volume (RVEDV), RVEDP, and right ventricular Ees, are shown in Figure 2 as a function of right ventricular afterload (RVSP). Before CPB and cardioplegic arrest, a moderate increase of right ventricular afterload (RVSP of approxi- 


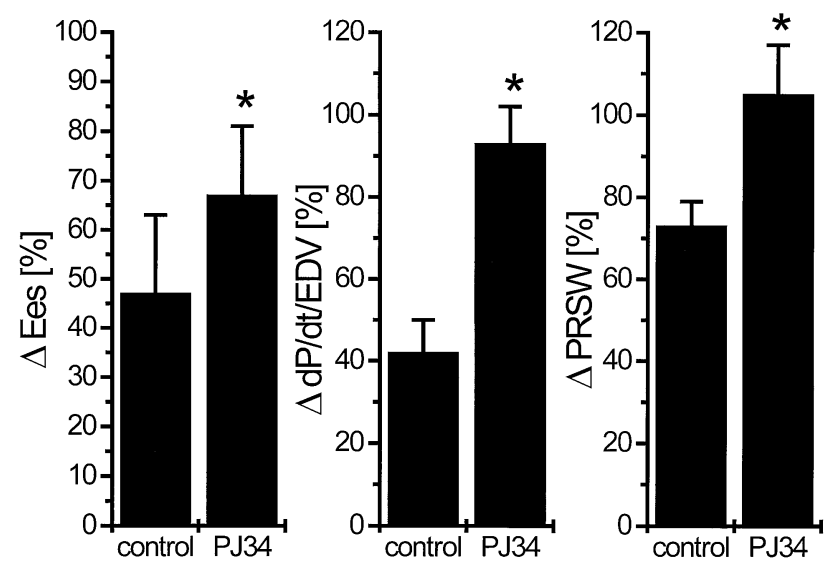

Figure 1. Percentage change of Ees, slope of the left ventricular $\mathrm{dP} / \mathrm{dt}_{\max }$-end-diastolic volume relationship, and preload recruitable stroke work after CPB at 60 minutes of reperfusion. All values are given as means \pm SEM. ${ }^{*} P<.05$, PJ34 versus control.

mately $35 \mathrm{~mm} \mathrm{Hg}$ ) resulted in an isolated increase $(P<.05)$ in right ventricular Ees. A severe increase of right ventricular afterload (RVSP of approximately $55 \mathrm{~mm} \mathrm{Hg}$ ) led to a further increase $(P<.05)$ of right ventricular Ees, with a concomitant increase of RVESV, RVEDV, and RVEDP that did not reach the level of significance.

After 60 minutes of reperfusion, RVSP was slightly increased in the control group without reaching the level of significance. RVESV, RVEDV, and RVEDP did not differ in comparison with pre-CPB values, and right ventricular Ees showed a moderate decrease in the control group. An increase in right ventricular afterload led to a significant $(P<.05)$ increase in RVESV, RVEDV, and RVEDP, whereas the right ventricular Ees was significantly $(P<.05)$ lower in comparison with values before CPB at each level of pulmonary banding (Figure 2). In contrast, RVESV, RVEDV, and RVEDP, as well as right ventricular Ees, were nearly identical to pre-CPB values at baseline, and both levels of RV afterload increase in the PJ34 group.

\section{Coronary Vascular Function}

Coronary blood flow was similar in both groups before cardioplegic arrest. After 60 minutes of reperfusion, coronary blood flow decreased significantly $(P<.05)$ in the control group, whereas it remained unchanged in the PJ34 group (Figure 3). Endothelium-dependent and endotheliumindependent vasodilatation are shown in Figure 4. Endothelium-dependent vasodilatation after acetylcholine administration was significantly $(P<.05)$ reduced in both groups after 60 minutes of reperfusion in comparison with pre-CPB values. However, this decrease was significantly $(P<.05)$ smaller in the PJ34 group. Endothelium-independent vasodilatation after sodium nitroprusside showed no significant differences over time and between groups.

\section{Discussion}

In this study the benefits of the application of the novel PARP inhibitor PJ34 during reperfusion were assessed after cardioplegic arrest in a canine model of CPB. In accordance with the literature, $1,2,6,19,20$ hypothermic cardioplegic arrest and reperfusion resulted in a decrease in biventricular contractile and endothelial function. We showed, for the first time in a clinically relevant large-animal model, that the inhibition of PARP with PJ34 prevents biventricular dysfunction and improves endothelial recovery.

\section{Effects of PARP Inhibition}

The mechanisms of PJ34's protective action are multiple. Ischemia-reperfusion injury initiates a pathophysiologic cascade, including an inflammatory response with liberation of cytokines and free radicals. Triggered by means of peroxynitrite-induced DNA single-strand breaks, PARP catalyzes an energy-consuming polymerization of adenosine diphosphate-ribose, resulting in $\mathrm{NAD}^{+}$depletion, inhibition of glycolysis and mitochondrial respiration, and reduction of intracellular high-energy phosphates in the reperfused heart. ${ }^{13,14,21,22}$ The recently discovered mechanism of cell injury, the PARP pathway, ${ }^{21,22}$ is involved in the pathogenesis of various forms of ischemia-reperfusion injury. We showed previously in a rat transplant model of hypothermic ischemia and reperfusion ${ }^{18}$ that the prevention of PARP activation by PJ34 results in a better preservation of the total adenylate pool, primarily by means of the increased myocardial adenosine triphosphate content, resulting in an improved energy status.

In addition to its direct effects on myocardial metabolism, PARP activation contributes to the expression of Pselectin and intercellular adhesion molecule 1 (ICAM-1) during cardiac ischemia and reperfusion ${ }^{9,18}$ and, consequently, to the recruitment of neutrophils into jeopardized tissue. ${ }^{23,24}$ We have previously reported ${ }^{9}$ that genetic disruption of PARP abolished the expression of P-selectin and the upregulation of ICAM-1 while maintaining unaffected constitutive levels of ICAM-1 on endothelial cells after 1 hour of coronary occlusion and 1 hour of reperfusion in a mouse model. The results of our rat heart transplant study ${ }^{18}$ clearly demonstrated that the inhibition of PARP activity can interrupt the interaction between neutrophils and endothelial cells both at the early rolling phase mediated by P-selectin and at the late firm adhesion phase mediated by ICAM-1.

\section{Left and Right Ventricular Function}

The prevention of left and right ventricular contractile dysfunction by means of PARP inhibition is in accordance with results found in previous literature. In 1997, Thiemermann and colleagues ${ }^{13}$ and Zingarelli and associates ${ }^{14}$ independently demonstrated that pharmacologic inhibition of PARP reduces 

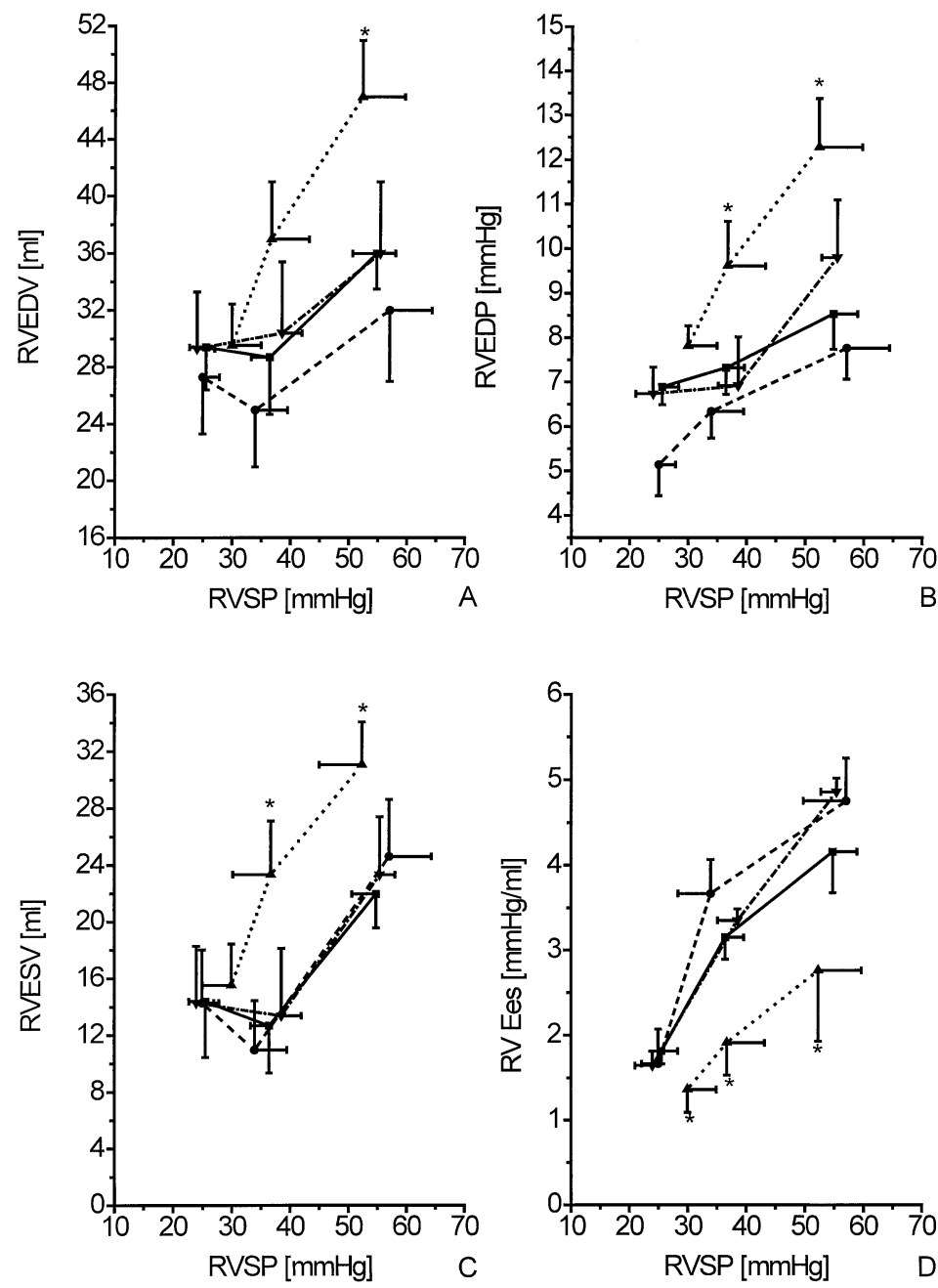

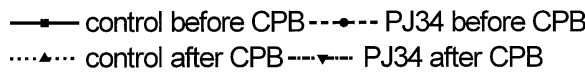

Figure 2. RVEDV (A), RVEDP (B), RVESV (C), and slope of right ventrciular Ees (RV Ees; D) as a function of RVSP before and after CPB at 60 minutes of reperfusion. All values are given as means \pm SEM. ${ }^{*} P<.05$ versus other runs.

myocardial necrosis and improves cardiac function in coronary ischemia-reperfusion injury. In addition, the beneficial effects of PARP deficiency ${ }^{25}$ or PARP inhibitors ${ }^{26}$ on functional contractile parameters ${ }^{25,26}$ and on high-energy phosphates ${ }^{26}$ after global ischemia and reperfusion of the heart have been reported. We demonstrated that PARP inhibition results in a complete recovery of left ventricular contractility after cardiac preservation and reperfusion. ${ }^{18}$

Because right ventricular afterload increase is a common condition after cardiac surgery with $\mathrm{CPB},,^{2-5}$ special emphasis was focused on the adaptation potential of the right ventricle to an increased afterload. In previous studies 27,28 it was demonstrated that an isolated increase of right ventric-

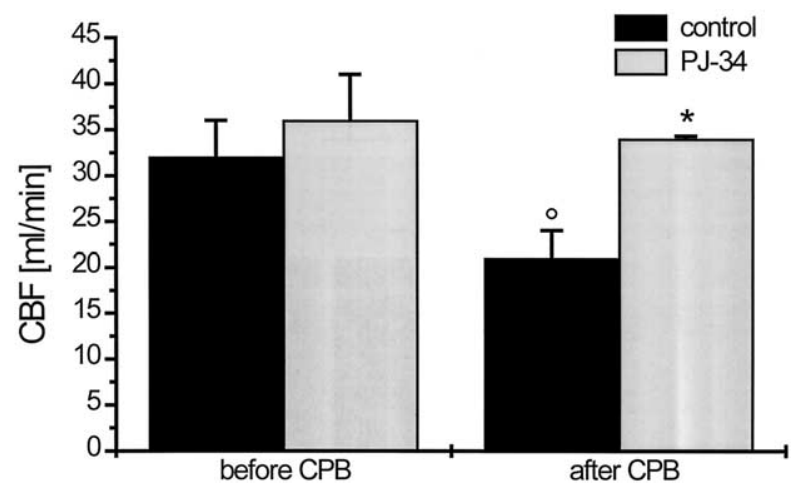

Figure 3. Coronary blood flow before and after CPB at 60 minutes of reperfusion. All values are given as means \pm SEM. ${ }^{\circ} P<.05$ versus baseline; ${ }^{*} \boldsymbol{P}<.05$ PJ34 versus control. 


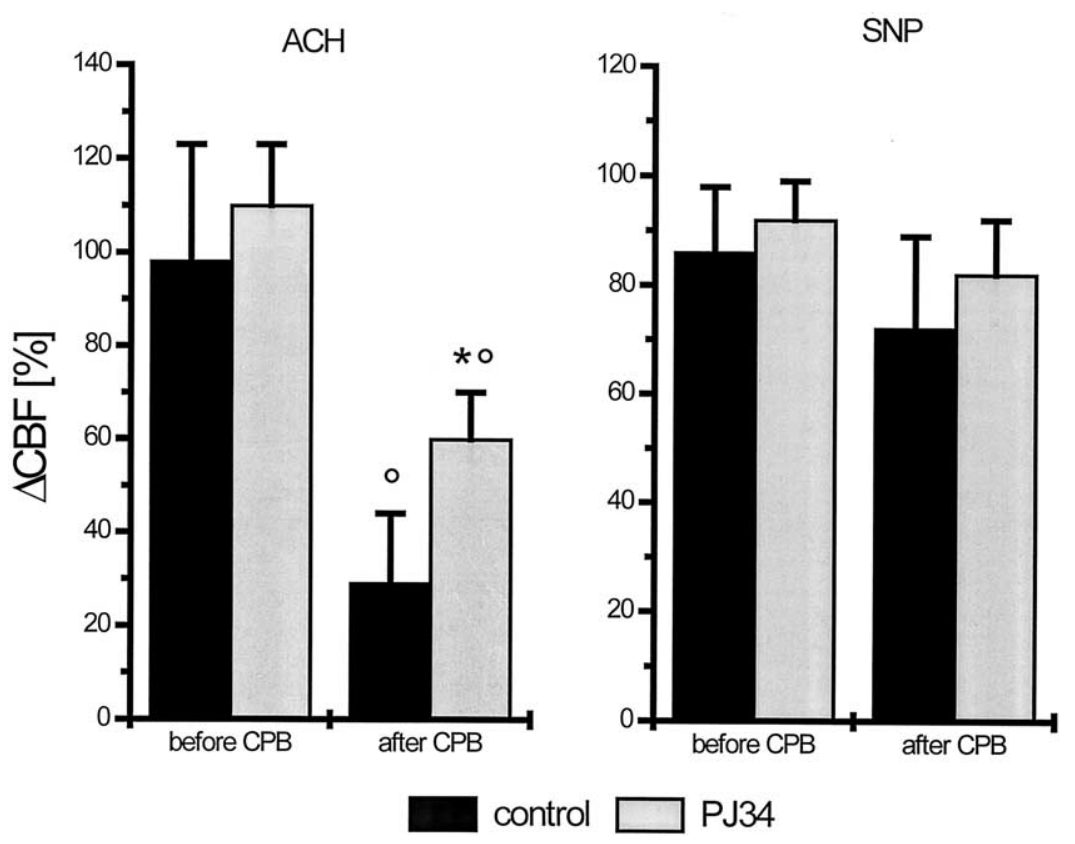

Figure 4. Coronary vascular function. Endothelium-dependent vasodilatation after acetylcholine $\left(\mathrm{ACH} ; \mathbf{1 0}^{-7} \mathrm{~mol} / \mathrm{L}\right.$, left pane ) and endothelium-independent vasodilatation after sodium nitroprusside (SNP, $10^{-4} \mathrm{~mol} / \mathrm{L}$; right panel). All values are given as means \pm SEM. ${ }^{\circ} P<.05$ versus baseline; ${ }^{*} P<.05$, PJ34 versus control.

ular afterload leads to an increase of myocardial contractility (homeometric autoregulation). In damaged hearts the heterometric autoregulation (ie, increase of preload) was the primary compensatory mechanism to maintain physiologic cardiac output values. ${ }^{27}$

The present data confirm previous observations that the increase of afterload resulted in a compensatory increase of contractility (right ventricular Ees). Even a severe increase of right ventricular afterload led only to a tendency toward higher right ventricular volumes without reaching the level of significance, indicating that inotropic adaptation is the primary compensatory mechanism of the right ventricle to an increased afterload. In contrast, after hypothermic cardiac arrest and reperfusion, an increase of right ventricular afterload resulted in a progressive increase of RVEDV and RVEDP, whereas right ventricular Ees showed only a slight increase. This indicates the use of the Frank-Starling mechanism (heterometric autoregulation) as a primary form of adaptation to increased afterload while inotropic adaptation remains limited.

PARP inhibition completely abolished the detrimental effects of cardioplegic arrest and reperfusion on right ventricular inotropic adaptation potential, probably as a result of the preservation of energy reserves.

How far increased right coronary perfusion pressure in the PJ34 group (as a consequence of increased blood pressure) influences right ventricular contractility remains unknown. There are only very few data available, even under physiologic conditions, that describe the effect of coronary perfusion pressure on right ventricular function, and the results are controversial. Unfortunately, we also do not have any evidence at this point. However, the differences in blood pressure between the groups were rather low to explain the observed differences in right ventricular contractility.

\section{Coronary Vascular Function}

According to the literature, ${ }^{6,20,29}$ coronary blood flow was reduced, and endothelial function was impaired, whereas smooth muscle function remained unaffected ${ }^{6,29-31}$ after hypothermic cardiac arrest and reperfusion. Potential mechanisms include free radical-mediated cytotoxicity, ${ }^{6,20}$ neutrophil-endothelium interactions, ${ }^{18,29}$ and impaired nitric oxide synthesis. ${ }^{29}$ Although PJ34 does not have its own vasodilatory property, ${ }^{18}$ PARP inhibition completely prevented the decrease of coronary blood flow and significantly improved endothelial function, which was comparable with that seen in other studies with nitric oxide donors ${ }^{29-31}$ or free radical scavengers. ${ }^{6}$ There is strong evidence that free radical production and oxidative stress are major contributing factors to endothelial dysfunction after cardioplegic arrest and reperfusion. ${ }^{6}$ It was also shown that energy depletion mediated by PARP after oxidant stress significantly contributes to endothelial injury in cultured pulmonary artery endothelial cells, in endotoxin shock, and also in diabetes mellitus in vivo, as well as in rat cardiac transplants. ${ }^{32-34}$ Under these 
aspects, the observed beneficial effects of PJ34 are most likely related to the prevention of energy depletion of endothelial cells. Furthermore, the abovementioned inhibition of P-selectin and ICAM-1 might also contribute to reduced endothelial injury in the PJ34 group.

The fact that contractile function and coronary blood flow were completely restored and endothelial function was still impaired in the PJ34 group indicates that either other mechanisms than those leading to PARP activation are also involved in endothelial injury after hypothermic cardiac arrest or that the endothelium is more vulnerable to the ischemic stimulus. ${ }^{18,29}$

In summary, we demonstrated that hypothermic cardiac arrest followed by reperfusion leads to a significant impairment of myocardial and endothelial function. In the present study a potent PARP inhibitor was able to markedly attenuate reperfusion injury, resulting in better functional recovery in a clinically relevant large animal model. On the basis of the present data and data from our previous studies, ${ }^{18}$ clinical studies with PARP inhibitors are warranted to reduce reperfusion injury and to improve postoperative cardiac function.

\section{References}

1. Wallace A, Lam HW, Nose PS, Bellows W, Mangano DT. Changes in systolic and diastolic ventricular function with cold cardioplegic arrest in man. The Multicenter Study of Perioperative Ischemia (McSPI) Research Group. J Card Surg. 1994;9(suppl 3):497-502.

2. Boldt J, Kling D, Moosdorf R, Hempelmann G. Influence of acute volume loading on right ventricular function after cardiopulmonary bypass. Crit Care Med. 1989;17:518-22.

3. Riedel B. The pathophysiology and management of perioperative pulmonary hypertension with specific emphasis on the period following cardiac surgery. Int Anesthesiol Clin. 1999;37:55-79.

4. Morita K, Ihnken K, Buckberg GD, Sherman MP, Ignarro LJ. Pulmonary vasoconstriction due to impaired nitric oxide production after cardiopulmonary bypass. Ann Thorac Surg. 1996;61:1775-80.

5. Cave AC, Manche A, Derias NW, Hearse DJ. Thromboxane A2 mediates pulmonary hypertension after cardiopulmonary bypass in the rabbit. J Thorac Cardiovasc Surg. 1993;106:959-67.

6. Sellke FW, Shafique T, Ely DL, Weintraub RM. Coronary endothelial injury after cardiopulmonary bypass and ischemic cardioplegia is mediated by oxygen-derived free radicals. Circulation. 1993;88:II395-400.

7. Heller B, Wang ZQ, Wagner EF, Radons J, Burkl A, Fehsel K, et al. Inactivation of the poly (ADP-ribose) polymerase gene affects oxygen radical and nitric oxide toxicity in islet cells. J Biol Chem. 1995;270: 11176-80.

8. Virág L, Scott GS, Cuzzocrea S, Salzman AL, Szabó C. Peroxynitriteinduced thymocyte apoptosis: the role of caspases and poly-(ADPribose) synthetase (PARS) activation. Immunology. 1998;94:345-55.

9. Zingarelli B, Salzman AL, Szabó C. Genetic disruption of poly(ADPribose) synthetase inhibits the expression of P-selectin and intracellular adhesion molecule-1 in myocardial ischemia/reperfusion injury. Circ Res. 1998;83:85-97.

10. Szabó C, Zingarelli B, O'Connor M, Salzman AL. DNA strand breakage, activation of poly-ADP ribosyl synthase, and cellular energy depletion are involved in the cytotoxicity in macrophages and smooth muscle cells exposed to peroxynitrite. Proc Natl Acad Sci U S A. 1996;93:1753-8.

11. Bowes J, McDonald MC, Piper J, Thiemermann C. Inhibitors of poly (ADP-ribose) synthetase protects rat cardiomyocytes against oxidant stress. Cardiovasc Res. 1999;41:126-34.
12. Szabó C, Dawson VL. Role of poly (ADP ribose) synthetase activation in inflammation and reperfusion injury. Trends Pharmacol Sci. 1998;19:287-98.

13. Thiemermann C, Bowes J, Myint FP, Vane JR. Inhibition of the activity of poly (ADP ribose) synthetase reduces ischemia-reperfusion injury in the heart and skeletal muscle. Proc Natl Acad Sci U S A. 1997;94:679-83.

14. Zingarelli B, Cuzzocrea S, Zsengellér Zs, Salzman AL, Szabó C. Protection against myocardial ischemia and reperfusion injury by 3-aminobenzamide, an inhibitor of poly (ADP ribose) synthetase. Cardiovasc Res. 1997;36:205-15.

15. Liaudet L, Yang Z, Al-Affar EB, Szabó C. Myocardial ischemic preconditioning in rodents is dependent on poly (ADP-ribose) synthetase. Mol Med. 2001;7:406-17.

16. Szabó C, Cuzzocrea S, Zingarelli B, O’Connor M, Salzman AL. Endothelial dysfunction in a rat model of endotoxin shock. Importance of the activation of poly (ADP-ribose) synthetase by peroxynitrite. J Clin Invest. 1997;100:723-35.

17. Cuzzocrea S, Zingarelli B, Constantino G, Szabó A, Salzmann AL, Caputi AP, et al. Beneficial effects of 3-aminobenzamide, an inhibitor of poly (ADP-ribose) synthetase in a rat model of splanchnic artery occlusion and reperfusion. Br J Pharmacol. 1997;121:1065-74.

18. Szabó G, Bährle S, Stumpf N, Sonnenberg K, Szabó E, Pacher P, et al. Poly (ADP-ribose) polymerase inhibition reduces reperfusion injury after heart transplantation. Circ Res. 2002;90:100-6

19. Gorcsan J 3rd, Gasior TA, Mandarino WA, Deneault LG, Hattler BG, Pinsky MR. Assessment of the immediate effects of cardiopulmonary bypass on left ventricular performance by on-line pressure-area relations. Circulation. 1994;89:180-90.

20. Aoki M, Kawata H, Mayer JE Jr. Coronary endothelial injury by cold crystalloid cardioplegic solution in neonatal lambs. Circulation. 1992; 86:II346-51.

21. Sims JL, Berger SJ, Berger NA. Poly (ADP-ribose) Polymerase inhibitors preserve nicotinamide adenine dinucleotide and adenosine 5 '-triphosphate pools in DNA-damaged cells: mechanism of stimulation of unscheduled DNA synthesis. Biochemistry. 1983;22:5188-94.

22. Schraufstatter IU, Hinshaw DB, Hyslop PA, Spragg RG, Cochrane CG. Oxidant injury of cells. DNA strand breaks activate polyadenosine diphosphate-ribose polymerase and lead to depletion of nicotinamide adenine dinucleotide. J Clin Invest. 1986;77:1312-20.

23. Jerome SH, Doré M, Paulson JC, Smith CW, Korthuis RJ. P-Selectin and ICAM-1-dependent adherence reactions: role in the genesis of postischemic no-reflow. Am J Physiol. 1994;266:H622-30.

24. Weyrich AS, Ma XL, Lefer DJ, Albertine KH, Lefer AM. In vivo neutralization of P-selectin protects feline heart and endothelium in myocardial ischemia and reperfusion injury. J Clin Invest. 1993;91: 2620-9.

25. Grupp IL, Jackson TM, Hake P, Grupp G, Szabó C. Protection against hypoxia-reoxygenation in the absence of poly (ADP-ribose) synthetase in isolated working hearts. J Mol Cell Cardiol. 1999;31:297303.

26. Docherty JC, Kuzio B, Silvester JA, Bowes J, Thiemermann C. An inhibitor of poly (ADP-ribose) synthetase activity reduces contractile dysfunction and preserves high energy phosphate levels during reperfusion of the ischemic rat heart. Br J Pharmacol. 1999;127: 1518-24.

27. Szabó G, Sebening C, Hagl C, Tochtermann U, Vahl CF, Hagl S. Right ventricular function after brain death: response to an increased afterload. Eur J Cardiothorac Surg. 1998;13:449-59.

28. De Vroomen M, Lopes Cardoso RH, Steendijk P, Van Bel F, Baan J. Improved contractile performance of right ventricle in response to increased RV afterload in newborn lamb. Am J Physiol. 2000;278: H100-5.

29. Szabó G, Bährle S, Bátkai S, Stumpf N, Dengler TJ, Vahl CF, et al. L-arginine: effect on reperfusion injury after heart transplantation. World J Surg. 1998;22:791-8.

30. Lefer DJ, Nakanishi K, Vinten-Johansen J. Endothelial and myocardial cell protection by a cysteine-containing nitric oxide donor after myocardial ischemia and reperfusion. J Cardiovasc Pharmacol. 1993; 22(suppl 7):S34-43. 
31. Sato H, Zhao ZQ, McGee DS, Williams MW, Hammon JW Jr, Vinten-Johansen J. Supplemental L-arginine during cardioplegic arrest and reperfusion avoids regional postischemic injury. J Thorac Cardiovasc Surg. 1995;110:302-14.

32. Soriano FG, Virág L, Jagtap P, Szabó É, Mabley JG, Liaudet L, et al. Diabetic endothelial dysfunction: the role of poly (ADP-ribose) polymerase activation. Nat Med. 2001;7:108-13.

33. Thies RL, Autor AP. Reactive oxygen injury to cultured pulmonary artery endothelial cells: mediation by poly ADP-ribose polymerase activation causing NAD depletion and altered energy balance. Arch Biochem Biophys. 1991;286:353-63.

34. Szabó C, Lim LH, Cuzzocrea S, Getting SJ, Zingarelli B, Flower RJ, et al. Inhibition of poly (ADP-ribose) synthetase exerts anti-inflammatory effects and inhibits neutrophil recruitment. J Exp Med. 1997; 186:1041-9.

\section{Discussion}

Dr Frank W. Sellke (Boston, Mass). Do you think most of the injury that PARP inhibited was reducing oxidative stress?

Dr Szabó. We have previous data that show that PARP inhibition per se does not reduce oxidative stress, but the consequences are reduced. A major advantage of this concept is that PARP inhibition stops the process induced by free radicals at the most distal point. There are numerous pathways that induce free radical production and its subsequent steps. Even if there are many experimental studies with radical scavengers or anti-inflammatory agents, they mostly fail under the clinical situation because there are a lot of parallel pathways. We think PARP inhibition can prevent the cumulative effects of these parallel pathways at the cell nuclear level

Dr Sellke. Did you look at nitrotyrosine levels?

Dr Szabó. We did not in the present study. We published a study with rat heart transplants in Circulation Research in January 2002 with detailed biochemical and immunohistologic investigations. We observed nitrotyrosine positivity after hypothermic cardiac arrest either with or without PARP inhibition.

Dr Sellke. With cardiac surgery, you can decide for yourself when to administer the drug. Why did not you administer it before onset of bypass or onset of cardioplegia, or perhaps even put it in the cardioplegic solution, rather than administering it with onset of reperfusion? It was obviously effective, but I would have thought that you could have optimized the benefit had you administered it a little bit earlier.

Dr Szabó. We have also performed studies to investigate when PARP activation occurs. We stained poly-ADP-ribose, the product of PARP. We could show PARP activation, of course, during reperfusion but not during the ischemic period. A recent study from another group has even shown that PARP activation lasts up to 1 to 3 days in the brain, for example. Therefore PARP inhibition might even be a good therapeutic option for rescue therapy 4 or 5 hours after the insult. 